\title{
Anastomosis intestinal sin sutura. Uso de adhesivos sintéticos en un modelo experimental
}

\author{
Flores-Álvarez Efrén*, Alvarado-Murillo Ramón Fernando**, \\ De la Torre-González José Cruz***
}

\begin{tabular}{|c|c|}
\hline $\begin{array}{l}\text { Resumen } \\
\text { El uso de adhesivos sintéticos ha sido investigado en diversas } \\
\text { áreas quirúrgicas, en cirugía gastrointestinal parecen tener } \\
\text { utilidad. Se han reportado estudios experimentales que de- } \\
\text { muestran que es posible llevar a cabo anastomosis sin su- } \\
\text { tura. Objetivo: Demostrar la utilidad de los sellantes tisulares } \\
\text { sintéticos sin el uso de material de sutura como alternativa } \\
\text { en la realización de anastomosis intestinales en animales de } \\
\text { experimentación. Material y métodos: Estudio experimen- } \\
\text { tal en conejos Rex albino, fueron incluidos } 3 \text { grupos de lo } \\
\text { conejos cada uno. En el grupo A (grupo control), se realizó } \\
\text { una anastomosis a nivel del colon transverso con material de } \\
\text { sutura convencional; en el grupo B (grupo Dermabond), una } \\
\text { anastomosis empleando adhesivo sintético 2-octil cianocrilato, } \\
\text { y en el grupo C (grupo Coseal), una anastomosis con aplica- } \\
\text { ción del adhesivo sintético Coseal. Al quinto día se realizó una } \\
\text { laparotomía exploradora para evaluar las condiciones de las } \\
\text { anastomosis colónicas. Resultados: Al evaluar la dehiscencia } \\
\text { de la anastomosis colónica se observó que estuvo presente en } \\
\text { dos conejos del grupo control, en cinco de los conejos del gru- } \\
\text { po Dermabond y todos los del grupo Coseal (p=0.003). De } \\
\text { las complicaciones que con mayor frecuencia se reportaron } \\
\text { asociadas a la presencia de dehiscencia de la anastomosis } \\
\text { fueron las adherencias posoperatorias y los abscesos peria- } \\
\text { nastomóticos. Conclusión: El uso de adhesivos sintéticos como } \\
\text { medio de unión en las anastomosis intestinales colónicas en } \\
\text { conejo Rex no es seguro ni eficaz por el alto porcentaje de } \\
\text { dehiscencia de la anastomosis. Lux méDICA AÑo 8, NúmER0 25, } \\
\text { SEPTIEmBRE-DicIEmBRE 2013, PP 3-9 }\end{array}$ & 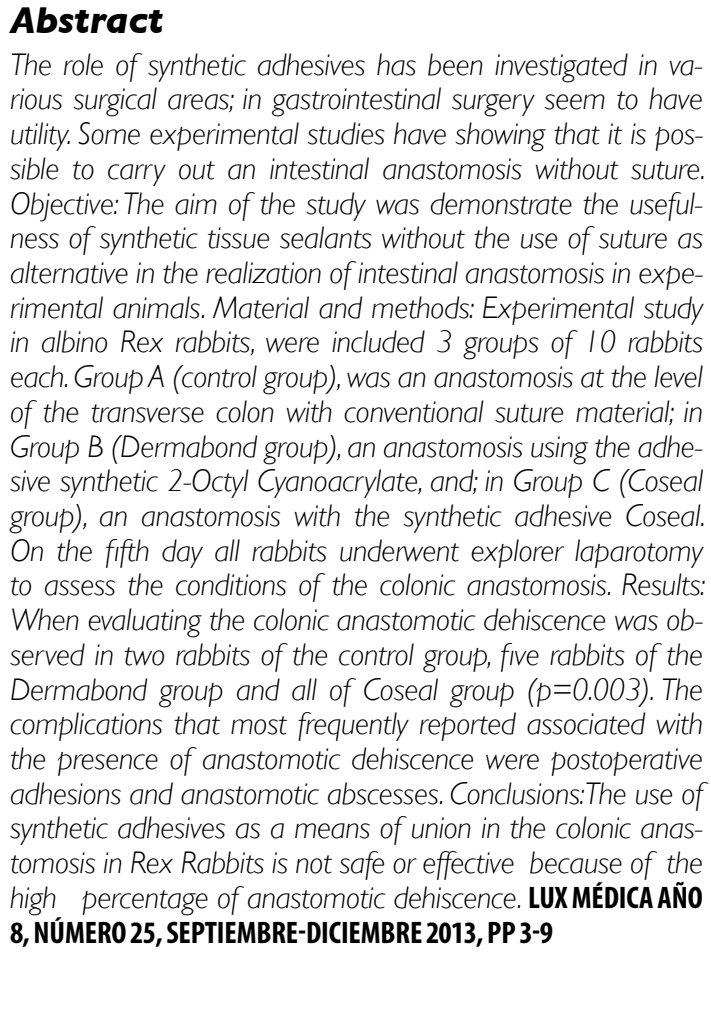 \\
\hline $\begin{array}{r}\text { Palabras clave: Ana } \\
\text { sintétic }\end{array}$ & astomosis, synthetic adhesives, \\
\hline
\end{tabular}

\footnotetext{
Maestro en Ciencias Médicas, Profesor titular de posgrado en Cirugía General, Centenario Hospital Miguel Hidalgo.

** Médico Residente de Cirugía General, Centenario Hospital Miguel Hidalgo.

*** Profesor adjunto de Cirugía General, Centenario Hospital Miguel Hidalgo.

Fecha de recibido: 1 agosto 2013

Fecha de aceptación: 9 de octubre 2013

Correspondencia: MCM Efrén Flores Álvarez. Maestro en Ciencias Médicas, Profesor titular de posgrado en Cirugía General del Centenario Hospital Miguel Hidalgo. Calle Galeana Sur 3465 Colonia Obraje CP 20230, Aguascalientes, Ags., México Teléfono 01 (449) 9946720 Correo electrónico
} efflorez@hotmail.com 


\section{Introducción}

A pesar de los avances tecnológicos y un mayor conocimiento de los mecanismos biomoleculares involucrados en los procesos de cicatrización de las anastomosis intestinales, la dehiscencia continúa siendo una temida complicación que se presenta con relativa frecuencia en la práctica clínica de la cirugía gastrointestinal. Se reportan tasas de dehiscencia de anastomosis intestinales tan alta como del 10 al 50\% con el uso de sutura convencional. ${ }^{1,2}$

Son múltiples los factores de riesgo conocidos que influyen en la adecuada cicatrización de una anastomosis intestinal: la edad del paciente, el estado nutricional, la presencia de procesos neoplásicos, la técnica empleada, la experiencia del cirujano y el material de sutura. ${ }^{3}$ Los factores técnicos más importantes en el éxito de una anastomosis intestinal son la experiencia del cirujano, el tipo de material de sutura y la técnica quirúrgica utilizada. Para llevar a buen término una anastomosis deben cumplirse las siguientes premisas: (1) colocación de suficiente número de puntos poco separados uno de otro, (2) invertir la mucosa para evitar que sobresalga por la línea de sutura, (3) poner frente a frente las superficies serosas para formar una unión sólida lo más rápido posible, (4) cohibir la hemorragia de la pared intestinal en forma precisa, (5) aproximar los bordes seccionados de la mucosa para evitar una estenosis cicatricial, (6) evitar la tensión en la línea de sutura y (7) el cierre o las anastomosis deben realizarse siempre en bordes con buena irrigación sanguínea. ${ }^{4}$

El material ideal de sutura para efectuar una anastomosis es aquel que conserva su resistencia hasta la plena integridad de la misma y asegura el proceso de cicatrización completo, causa una mínima reacción tisular, sea resistente a la infección y sea de fácil manipulación. En la actualidad continúan en debate los diferentes métodos empleados para crear una anastomosis, debido a que no se cuenta con estudios clínicos con evidencia contundente que indiquen que una técnica determinada tiene ventajas sobre las demás. Algunos autores han tratado de mejorar las condiciones locales o sistémicas para mejorar los resultados de las anastomosis ${ }^{5-7}$. Diversos estudios han demostrado mejoría en el proceso de cicatrización de las anastomosis mediante la aplicación de nuevos métodos de sutura, refuerzos o parches en la línea de la anastomosis, sin embargo la mayoría de estos estudios no han podido ser reproducidos en otros sitios y en algunas ocasiones se ha demostrado su ineficacia en la práctica clínica. Dentro de estas alternativas estudiadas se encuentran las sustancias adhesivas, las cuales actúan mediante un mecanismo de polimerización al contacto con los tejidos, dicha polimerización une los extremos de los segmentos intestinales llevando a cabo la anastomosis, además actúa como sellante para prevenir las fugas anastomóticas ${ }^{8-10}$.

El objetivo del presente estudio es demostrar la utilidad de los sellantes tisulares sintéticos sin el uso de material de sutura como alternativa en la realización de anastomosis intestinales en animales de experimentación. 


\section{Material y métodos}

Se realizó un estudio experimental, prospectivo, comparativo y transversal en conejos Rex albino; se estudiaron tres grupos de 10 conejos cada uno. Fueron incluidos conejos hembras, clínicamente sanos, de entre 20 y 45 días de edad, con un peso entre 400 y $800 \mathrm{~g}$. Fueron manejados en el bioterio de la Universidad Autónoma de Aguascalientes de acuerdo a la Norma Oficial Mexicana (NOM-062-200-1999). El protocolo fue revisado y avalado por el comité local e institucional de ética e investigación. En el grupo $A$, grupo control, se realizó una anastomosis a nivel del colon transverso, con material de sutura monofilamento no absorbible $6 / 0$, con puntos totales separados (imagen 1); en el grupo $B$, grupo Dermabond, se efectuó una anastomosis del colon transverso aplicando adhesivo sintético 2-octil cianocrilato (Dermabond) como medio de unión, con dos puntos cardinales de fijación con monofilamento no absorbible 6/0 (imagen 2), y en el grupo $C$, grupo Coseal, se realizó una anastomosis del colon transverso con aplicación del adhesivo sintético Coseal, con dos puntos cardinales de fijación con monofilamento no absorbible 6/0 (imagen 3). Al quinto día, se llevó a cabo una laparotomía exploradora para revisar las condiciones de las anastomosis colónicas, además de medir la presión de estallamiento y resecar el segmento de intestino anastomosado para hacer un análisis histopatológico. Fueron excluidos aquellos conejos que murieron en la fase peri-operatoria y aquellos que presentaron complicaciones propias del procedimiento anestésico o quirúrgico que dificultaron la evaluación de las variables estudiadas.

Se realizó un análisis descriptivo de cada una de las variables estudiadas. En las variables cualitativas se calculó la frecuencia absoluta y el porcentaje. En las variables cuantitativas se estimó la mediana y los rangos, debido a que los datos mostraban una distribución no normal. Para demostrar diferencias entre los grupos se utilizó la prueba de chi cuadrada y la prueba $U$ de Mann-Withney de acuerdo al tipo de variable. Se consideró como significancia estadística cuando el valor de $\mathrm{p}$ fue menor de 0.05

\section{Resultados}

El grupo total de estudio estuvo integrado por 30 conejos de la raza Rex albino, todos hembras. El peso de los conejos pertenecientes al grupo control tuvo una mediana de 570 g. (rango de 400 a 600); los del grupo Dermabond, 555 g. (rango de 420 a 600), y los del grupo Coseal, 590 g. (rango de 500 a 780). No existió diferencia estadística al comprar el peso entre los 3 grupos $(p=0.87)$. La edad de los conejos en el grupo control tuvo una mediana de 25 días (rango de 20 a 27); los del grupo Dermabond, 25 días (rango de 18 a 30), y los del grupo Coseal, 28 días (rango de 21 a 34). Tampoco se observó diferencia estadística significativa $(p=0.7)$ (tabla 1$)$.
La duración del procedimiento quirúrgico tuvo una mediana de 49 minutos en el grupo control (rango de 38 a 73); de 2.05 minutos (rango de 1.48 a 3.05) en el grupo Dermabond, y de 2.09 minutos (rango de 1.5 a 2.48) en el grupo Coseal . La diferencia estadística fue altamente significativa al comparar los grupos $(p<0.001)$, en favor de los grupos de los adhesivos, en que el tiempo fue mucho menor.

Al evaluar la dehiscencia de la anastomosis colónica se observó que estuvo presente en dos conejos del grupo control (20\%), en cinco de los conejos (50\%) del grupo Dermabond y en todos los integrantes (100\%) del grupo Coseal, los cuales presentaron dehiscencia en un lapso no mayor a $48 \mathrm{hr}$ 
$(p=0.003)$ (tabla 2). De las complicaciones que con mayor frecuencia se reportaron asociadas a la presencia de dehiscencia de la anastomosis fueron las adherencias posoperatorias y los abscesos peri-anastomóticos.

La mediana de la presión de estallamiento en el grupo control fue de $39 \mathrm{~cm} \mathrm{H}_{2} \mathrm{O}$ (rango de 36 a 50) y en el grupo Dermabond, de $10 \mathrm{~cm} \mathrm{H}_{2} \mathrm{O}$ (rango de 2 a 33). No fue posible hacer un análisis estadístico comparativo respecto a la presión de estallamiento debido a que todos los conejos del grupo Coseal tuvieron dehiscencia de más del $80 \%$ de la circunferencia de la anastomosis.
El análisis histopatológico de las piezas quirúrgicas demostró la presencia de fibrosis en cantidad moderada a intensa en el grupo de sutura convencional, leve en el grupo de Dermabond y ausente en el grupo Coseal (tabla 3). También se observó una mayor respuesta inflamatoria local en los especímenes de los conejos tratados con adhesivos sintéticos indicado por la presencia de abundantes polimorfonucleares en las áreas adyacentes al sitio de la anastomosis. Es importante resaltar que en ninguno de los especímenes se identificó reacción inflamatoria a cuerpo extraño ni la presencia de granulomas.

| | | | | | | | | | | | | | | | | | | | | | | | | | | | | | | | | | | | | | | | | | | | | | | | | | | | | | | | | | | | | | | | | | | | | | | | | | | | | | | | | | | | | | | | | | | | | | | | | | |

\section{Discusión}

La anastomosis intestinal es un procedimiento ampliamente realizado en la práctica quirúrgica ante diversas circunstancias o entidades patológicas, tales como lesiones neoplásicas, en la enfermedad vascular mesentérica de tipo isquémica, en procesos infecciosos abdominales complicados, en lesiones intestinales de origen traumático y en cualquier proceso que ocasiona pérdida de la integridad de la pared intestinal. ${ }^{4}$

A pesar de los avances tecnológicos y un mayor conocimiento de los mecanismos biomoleculares involucrados en los procesos de cicatrización de las anastomosis intestinales, la dehiscencia continua siendo una temida complicación que se presenta con frecuencia en la práctica clínica de la cirugía del tracto digestivo. Con la finalidad de reducir al mínimo las dehiscencias de las anastomosis intestinales se han realizado múltiples estudios experimentales con diversas técnicas quirúrgicas en las cuales se han empleado nuevos materiales de sutura, adhesivos naturales a base de fibrina, adhesivos sintéticos y diversos tipos de grapas, con resultados poco consistentes por lo que se continua experimentando con nuevas técnicas quirúrgicas. ${ }^{8-12}$
El uso de adhesivos ha sido investigado en diversas áreas quirúrgicas, en cirugía gastrointestinal parecen tener utilidad. Los estudios iniciales reportan ventajas sobre los métodos convencionales reduciendo el tiempo operatorio, proporcionando una buena flexibilidad en los tejidos, una adecuada fuerza tensil, una hermeticidad confiable, no produce adherencias, tiene una baja toxicidad y una adecuada cicatrización anastomótica. ${ }^{13}$

Los sellos de fibrina a nivel de la anastomosis incrementan el plano de adherencia tisular disminuyendo la micro y macro hemorragia en la línea de sutura y a través del mismo mecanismo evitan el acúmulo de exudado local. Además, se ha observado que aumenta el depósito de hidroxiprolina en las anastomosis, indicando un incremento en la cantidad de colágena local y una cicatrización más efectiva. La matriz de fibrina facilita la rápida migración leucocitaria y de fibroblastos además de acelerar el proceso de angiogénesis, lo cual favorece el proceso de cicatrización en sus etapas iniciales..$^{9,14} \mathrm{El}$ Coseal es un agente sellador hemostático sintético, se emplea en la práctica quirúrgica para lograr una hemostasia adjunta mediante el sellado mecánico en las líneas de 
sutura de anastomosis vasculares periféricas; produce un sello inmediato, de tipo gel que se conserva intacto en el sitio de aplicación. ${ }^{15}$ El Dermabond es un adhesivo tópico, líquido, que contiene una formación monomérica (2-octil cianocrilato), se polimeriza rápidamente después de su aplicación y es capaz de mantener aproximados los bordes de la piel en incisiones quirúrgicas y laceraciones sencillas de origen traumático. Varios estudios experimentales han demostrado la utilidad del Dermabond como adyuvante a la sutura quirúrgica en anastomosis intestinales reduciendo el índice de dehiscencia y las complicaciones secundarias a la fuga anastomótica. ${ }^{16}$

Lago-Oliver J y $\operatorname{cols}^{17}$, en un estudio multicéntrico, prospectivo, aleatorizado y controlado demostraron de manera significativa que los adhesivos biológicos a base de fibrina son capaces de prevenir la fuga anastomótica en anastomosis del tubo digestivo de alto riesgo, reduciendo el riesgo de fuga un $61 \%$ y el porcentaje de reintervenciones.

Con la finalidad de evaluar nuevas técnicas quirúrgicas, Zilling TL y cols. ${ }^{18}$ realizaron anastomosis experimentales en intestino delgado de cerdos, compararon la sutura convencional, la sutura con grapas y los adhesivos biológicos (sello de fibrina).
Demostraron que es posible llevar a cabo anastomosis sin sutura, sin dehiscencia, sin datos histológicos de rechazo, aunque con menor resistencia al ser sometidas a presión. Nuestros resultados, a diferencia de lo reportado en dicho estudio demuestran que tanto el sello de fibrina como el adhesivo sintético no producen una anastomosis adecuada por sí mismos y su utilidad podría estar limitada al empleo como refuerzo a una línea de sutura convencional.

Para la realización del presente estudio consideramos que el conejo representaba un buen modelo experimental por la factibilidad y experiencia en el manejo de estos animales en nuestro laboratorio, además de representar un modelo adecuadamente válido. Es cierto que no existen a la fecha estudios definitivos que apoyen el uso de adhesivos sintéticos y que aseguren la hermeticidad de una anastomosis con la menor lesión local y menores complicaciones propias de la anastomosis. Por ello se continúa estudiando las alternativas para asegurar la viabilidad en las anastomosis intestinales como lo fue el caso de este estudio experimental, en donde al emplear sellantes sintéticos de uso comercial se trató de demostrar su utilidad como medio de unión en la realización de una anastomosis intestinal comparándolos con las suturas convencionales.

\section{Conclusiones}

El uso de adhesivos sintéticos como medio de unión en las anastomosis colónicas en conejos Rex de experimentación no es eficaz y no es seguro sustituir el material se sutura convencional por adhesivos sintéticos ni biológicos. 


\section{Tabla I}

\section{Características generales de los conejos}

\begin{tabular}{|ccccc|}
\hline & Grupo control & Grupo Dermabond & Grupo Coseal & p \\
\hline $\begin{array}{c}\text { Sexo } \\
\text { Macho } \\
\text { Hembra }\end{array}$ & 0 & 0 & 0 & 1.0 \\
\hline $\begin{array}{c}\text { Edad (días) } \\
\text { Mediana }\end{array}$ & 10 & 10 & 10 & 0.74 \\
\hline $\begin{array}{c}\text { Peso (gramos) } \\
\text { Mediana }\end{array}$ & 25 & 25 & 28 & 0.87 \\
\hline
\end{tabular}

\section{Tabla 2}

\section{Dehiscencia de la anastomosis en los grupos}

\begin{tabular}{|c|ccc|c|}
\hline & Grupo Control & Grupo Dermabond & Grupo Coseal & Total \\
\hline Dehiscencia & & & & \\
Presente & 2 & 5 & 10 & 17 \\
Ausente & 8 & 5 & 0 & 13 \\
\hline Total & 10 & 10 & 10 & 30 \\
\hline
\end{tabular}

\section{Tabla 3}

Presencia de Fibrosis en los grupos

\begin{tabular}{|c|c|c|c|c|}
\hline & Grupo Control & Grupo Dermabond & Grupo Coseal & Total \\
\hline \multicolumn{5}{|l|}{ Fibrosis } \\
\hline Leve & 0 & 7 & 0 & 7 \\
\hline Moderado & 5 & 0 & 0 & 5 \\
\hline Intenso & 3 & 0 & 0 & 3 \\
\hline Ausente & 2 & 3 & 10 & 15 \\
\hline Total & 10 & 10 & 10 & 30 \\
\hline
\end{tabular}

$*$ Leve,+ Moderado ++ , Intenso +++ , Ausente 0 .

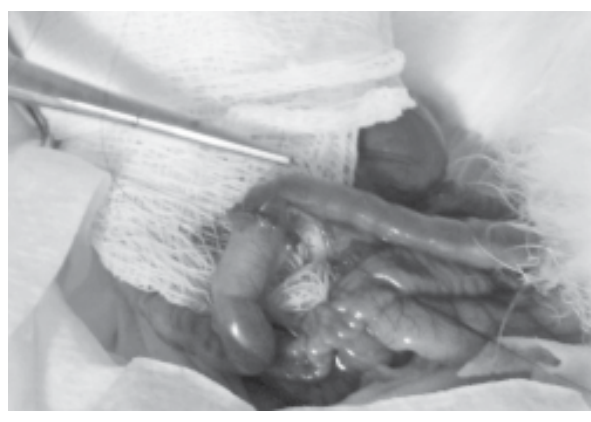

Imagen 1. Anastomosis colónica término- terminal, puntos separados con monofilamento $6 / 0$.

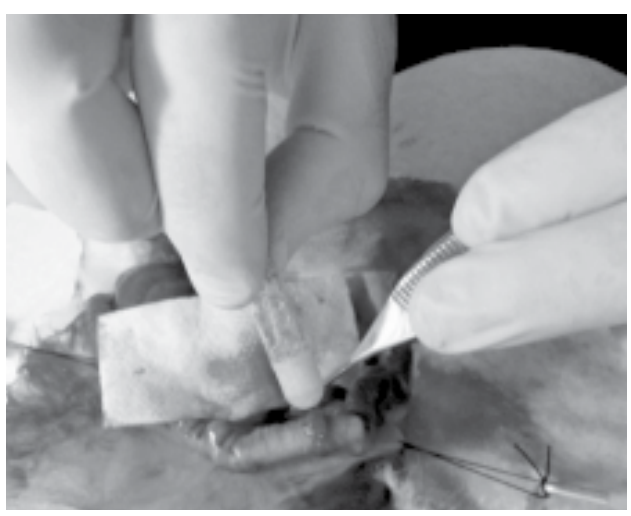

Imagen 2. Anastomosis colónica término- terminal con adhesivo Dermabond. 


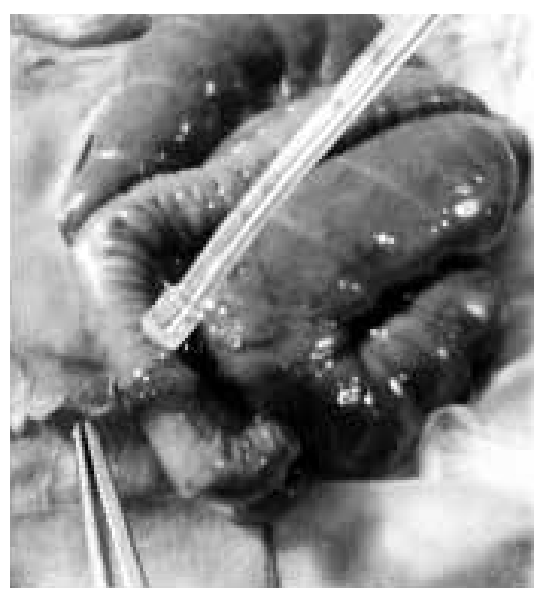

Imagen 3. Anastomosis colónica termino- terminal con adhesivo tisular

Coseal.

\section{Bibliografía}

1. Nandakumar G, Stein SL, Michelassi F. Anastomoses of the lower gastrointestinal tract. Nat Rev Gastroenterol Hepatol. 2009 Dec;6(12):709-16.

2. Pantelis D, Beissel A, Kahl P, Vilz TO, Stoffels B, Wehner S, Kalff JC. Colonic anastomotic healing in the context of altered macrophage function and endotoxemia. Int J Colorectal Dis. 2011 Jun;26(6):737-46.

3. Martínez JL, Luque-de-León E, Andrade P. Factors related to anastomotic dehiscence and mortality after terminal stomal closure in the management of patients with severe secondary peritonitis. J Gastrointest Surg. 2008 Dec;12(12):2110-8.

4. Flores AE, Montes de Oca VA, Rivera BV, Ornelas RE. Riesgo de dehiscencia de anastomosis de íleon terminal en conejo Rex albino tratados con infusión de noradrenalina. Cir Gen 2008; 30 (1):9-12.

5. Erturk S, Yuceyar S, Temiz M, Ekci B, Sakoglu N, Balci $H$, Dirican A, Cengiz A, Saner H. Effects of hyaluronic acid-carboxymethylcellulose antiadhesion barrier on ischemic colonic anastomosis: an experimental study. Dis Colon Rectum. 2003 Apr;46(4):529-34.

6. Yagci G, Ozturk E, Ozgurtas T, Gorgulu S, Kutlu OC, Topal T, Cetiner S, Tufan T. Preoperative and postoperative administration of hyperbaric oxygen improves biochemical and mechanical parameters on ischemic and normal colonic anastomoses. J Invest Surg. 2006 Jul-Aug;19(4):237-44.

7. Adas $G$, Karatepe $O$, Ar?kan S, Battal M, Kemik O, Altiok $M$, Kamali $G$, Karahan $S$. The effect of hyaluronic acid carboxymethyl cellulose on the healing of colonic anastomosis in rats. Bratisl Lek Listy. 2009;110(4):210-4.

8. Montanaro L, Arciola CR, Cenni E, Ciapetti G, Savioli F, Filippine F, Barsanti LA. Cytotoxicity, blood compartibility and antimicrobial activity of two cyanoac- rylate glues for surgical use. Biomaterials 2001; 22(1): 59-66.

9. Kanellos I, Mantzoros I, Goulimaris I et al. Effects of the use of fibrin glue around the colonic anastomosis of the rat. Tech Coloproctol 2003; 7(2): 82-84.

10. Matsumoto T. Ed. 1972. Tissue Adhesives in Surgery. Flushing, NY: Medical Examination Publishing Co.

11. Reece TB, Maxey TS, Kron IL. A prospectus on tissue adhesives. Am Journal of Surg. 2001; 182:40S-44S.

12. Gonzalez HD, Figueras Felip J. Topical hemostatic devices in surgery: between science and marketing. Cir Esp. 2009;85 Suppl. 1:23-8.

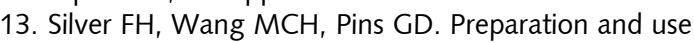
of fibrin glue in surgery. Biomaterial 1995; 16:891903.

14. Jackson MR, Macphee MJ, Drohann WN, Alving BM. Fribrin sealant: Current and potential clinical applications. Blood Coagul Fibrinolysis 1996;7:737-45.

15. COSEAL Surgical Sealant Instructions For Use, Hayward, CA: Baxter Healthcare Corporation. March 2009.

16. Singer AJ, Thode HC. A review of the literature on octyl cyanoacrylate tissue adhesive. Am Journal of Surg. 2004; 187:238-248.

17. Lago Oliver J, Arjona Medina I, Martín García-Almenta $E$, Martín Gil J, Sanz Sánchez M, Pérez Díaz MD, Alonso Poza A, Turégano Fuentes F, Torres García A. Utilización de adhesivos biológicos a base de fibrina en la prevención de fugas anastomóticas en anastomosis del tubo digestivo de alto riesgo: resultados preliminares del ensayo clínico fase IV multicéntrico, prospectivo, aleatorizado y controlado simple ciego: Protissucol001

Cir Esp. 2012 Dec;90(10):647-55.

18. Zilling $T L$, Jansson $O$, Walther BS, Ottosson A. Sutureless small bowel anastomoses: experimental study in pigs. Eur J Surg. 1999 Jan;165(1):61-8. 